\title{
Renal tumor biomarkers (Review)
}

\author{
OVIDIU BRATU ${ }^{1-3}$, DAN MISCHIANU ${ }^{1-3}$, DRAGOS MARCU $^{1,2}$, DAN SPINU $^{1,2}$, \\ LUCIAN IORGA $^{1,2}$, ALEXANDRU CHERCIU ${ }^{2}$, IRINA BALESCU ${ }^{4}$, NICOLAE BACALBASA $^{5-7}$, \\ CAMELIA DIACONU ${ }^{8,9}$, CORNEL SAVU $^{10,11}$, CARMEN SAVU $^{12}$ and RADU ANGHEL ${ }^{2}$
}

\begin{abstract}
${ }^{1}$ Department of Urology, 'Carol Davila' University of Medicine and Pharmacy, 020021 Bucharest; ${ }^{2}$ Department of Urology, University Emergency Central Military Hospital, 010825 Bucharest; ${ }^{3}$ Department of Urology,

Academy of Romanian Scientists, 020021 Bucharest; ${ }^{4}$ Department of Visceral Surgery, 'Ponderas' Academic Hospital, 021188 Bucharest; ${ }^{5}$ Department of Obstetrics and Gynecology, 'Carol Davila' University of Medicine and Pharmacy, 020021 Bucharest; ${ }^{6}$ Department of Visceral Surgery, Center of Excellence in Translational Medicine, 'Fundeni' Clinical Institute, 022328 Bucharest; ${ }^{7}$ Department of Obstetrics and Gynecology, 'I. Cantacuzino' Clinical Hospital, 030167 Bucharest; ${ }^{8}$ Department of Internal Medicine, 'Carol Davila' University of Medicine and Pharmacy, 020021 Bucharest; ${ }^{9}$ Department of Internal Medicine, Clinical Emergency Hospital of Bucharest, 105402 Bucharest; ${ }^{10}$ Department of Thoracic Surgery, 'Marius Nasta' National Institute of Pneumophtisiology, 050159 Bucharest; ${ }^{11}$ Department of Thoracic Surgery, 'Carol Davila' University of Medicine and Pharmacy, 020021 Bucharest; ${ }^{12}$ Department of Anesthesiology, 'Fundeni' Clinical Institute, 022328 Bucharest, Romania
\end{abstract}

Received May 5, 2021; Accepted June 4, 2021

DOI: $10.3892 / \mathrm{etm} .2021 .10732$

\begin{abstract}
One of the most common types of cancer worldwide (9th most commonly diagnosed) is renal cell carcinoma (RCC). It is more common in developed countries and it usually develops in individuals between 60 and 70 years of age. The earlier the disease is identified, the lower the morbidity. Therefore molecular markers that exist in blood and urine may be used for earlier detection and diagnosis but also for the follow-up of the patient after treatment, whether surgical or oncological. The trend is to analyze the gene and protein expression as they constitute a source for new biomarkers. These markers are promising but in clinical practice regarding disease management, they are rarely used. Biological markers can be employed in many tumors because they can identify the prognostic value for individual treatment. However, markers for RCC are not validated, and their analysis is currently
\end{abstract}

Correspondence to: Dr Nicolae Bacalbasa, Department of Obstetrics and Gynecology, 'Carol Davila' University of Medicine and Pharmacy, 37 Dionisie Lupu Street, 020021 Bucharest, Romania E-mail: nicolae_bacalbasa@yahoo.ro

Abbreviations: CA IX, carbonic anhydrase IX; CRP, C-reactive protein; GGT, $\gamma$-glutamyl transferase; HSP27, heat shock protein 27; NGAL, neutrophil gelatinase-associated lipocalin; RCC, renal cell carcinoma; TF, tissue factor; TK1, thymidine kinase 1; TRAF-1, tumor necrosis factor receptor-associated factor-1; TuM2 PK, pyruvate kinase type M2; VEGF, vascular endothelial growth factor

Key words: renal cell carcinoma, blood and urine biomarkers, circulating tumor cells, molecular markers, biological markers under investigation. Previous findings have demonstrated that the metastatic potential of RCC can be predicted using the biological features of the tumor cell. It is believed that the transformation from epithelial to mesenchymal phenotype gives the tumor cell the ability to metastasize. The purpose of this review was to identify the most valuable tumor markers that can be clinically used for the prognosis, treatment and follow-up of patients with renal tumors.

\section{Contents}

1. Introduction

2. Serum RCC biomarkers

3. Urine RCC biomarkers

4. Other useful biomarkers for RCC patients

5. Discussion

6. Conclusions

\section{Introduction}

One of the most common types of cancer worldwide (9th most commonly diagnosed) is renal cell carcinoma (RCC). It is more common in developed countries and it usually develops in individuals between 60 and 70 years of age (1). The earlier the disease is identified, the lower the morbidity. Therefore, molecular markers that exist in blood and urine may be used for earlier detection and diagnosis but also for the follow up of the patient after treatment, whether surgical or oncological (2). The trend is to analyze the gene and protein expression because they are a real source for new biomarkers. These markers have a promising potential but in clinical practice regarding 
the disease management, they are rarely used. The biological markers can be of use in many tumors because they can identify the prognostic for individual treatment, but the markers for RCC are not validated, and their analysis are currently under investigation (3). Previous findings have demonstrated that the metastatic potential of RCC can be predicted using the biological features of the tumor cell. It is believed that the transformation from epithelial to mesenchymal phenotype gives the tumor cell the ability to metastasize (4). The purpose of this review was to identify the best tumor markers that can be clinically used for the prognosis, treatment and follow-up of patients with renal tumors.

The highest incidence for renal tumors is represented by clear-cell renal carcinoma. In advanced stages, its morbidity and mortality are high. RCC is subclassified as clear cell, papillary and chromophobe. This is important because RCC is associated with different malignant potential. The clinical outcome of RCC is usually associated with the immune response. This type of disease is often diagnosed during a CT scan or renal echography for non-specific abdominal symptoms and often due to the fact that is mostly asymptomatic it is diagnosed in advanced stages; therefore, an early and efficient diagnostic technique is required. The principal prognostic factor for clinical work-up is represented by the histological analysis and the TNM stage and Fuhrman grade $(5,6)$. Previous findings showed a great prognostic value regarding tumor-infiltrating immune cells in the tumor to regulate cancer progression. The most studied type of cells is lymphocytes, tumor growth being prevented by the action of cytotoxic $\mathrm{CD}^{+} \mathrm{T}$ cells and $\mathrm{CD}^{+}$helper $\mathrm{T}$ cells $(5,6)$. Vascular endothelial growth factor (VEGF) and platelet-derived growth factor (PDGF) are recognized as valuable targets for targeted therapy (7). Currently, there are no available biomarkers for an accurate diagnosis of RCC. In certain studies, molecules that are measurable in serum have been reported (8). Immunological tests including ELISA or mass spectrometry have been used to identify altered proteins in RCC patients and can provide valuable information regarding prognosis and diagnosis. There is a protein linked to the proliferation, differentiation and even stress response (TRAF-1, tumor necrosis factor receptor-associated factor-1) that can be found in the serum of RCC patients (8).

\section{Serum RCC biomarkers}

CD105. A cell transmembrane glycoprotein known as CD105 has been found to be a key component in the transformation of growth factor $\beta$ receptor. It has been correlated with the tumor microvessel density and studies showed that CD105 can be a prognostic factor in RCC (9). Bussolati et al described this glycoprotein as a new RCC cancer stem cell marker (10). Saroufim et al performed a study where he evaluated 102 cases of partial/radical nephrectomy in patients with RCC, and the link between CD105 expression in endothelial and tumoral cells with stages, grades, and survival of these patients. Authors of that study found that CD105 had no correlation with nuclear grade and tumor stage in the endothelium, but CD105 tumor expression was positively correlated with these factors, and in conclusion this transmembrane glycoprotein self-determining predictive biomarker for death risk presents an unfavorable prognostic marker in patients with RCC (11).
HSP27/HSPB1. The study of Kampinga et al described a heat shock protein 27 (HSP27) or HSPB1 (12). This type of protein regulates different physiological processes of cells, being a small heat shock protein, in normal conditions, but has also been associated with tumor growth (13). The stress on the cell distributes HSP27 to the poles in order to regulate the dynamic structure of the cell. Stress also stimulates HSP27 depolymerization in small molecules, leading to polar actin accumulation and focal adhesion kinase, and eventually tumor cell migration (14). One of the main stress conditions is represented by hypoxia which leads to inflammation and oxidative stress, which in turn leads to tumor cell transformation and migration. The stress conditions determine this heat shock protein to be a part of the cellular stress response. Previous findings showed that HSP27 is hyperphosphorylated in many cancer types. For example, White et al described high levels of HSP27 in the serum and even in the urine of patients with high-grade renal tumors (15).

Pro-inflammatory cytokines and inflammatory immune cells. Inflammatory proteins including cytokines and inflammatory immune cells play a key role in kidney cancer owing to the constant search for new immunotherapeutic treatment of this disease. It is well known that some pro-inflammatory cytokines such as interleukin (IL)-1, IL-6, and tumor necrosis factor (TNF)- $\alpha$, in liver cells, can manage the synthesis of other inflammation factors such as amyloid A. Amyloid A then recruits immune cells to the inflammatory sites, and also produces enzymes that are involved in the destruction of the extracellular matrix (16). The identification of amyloid A in the serum of patients constitutes an important potential biomarker for patients with RCC, because, as indicated in other studies, it is also correlated with tumor stage especially advanced stages (17). In addition, acute-phase C-reactive protein (CRP) is a marker of advanced renal cancer, which can also be found in patients with this disease with a poor survival rate (17).

Another inflammatory protein and marker involved in human cancer is represented by the enzyme $\gamma$-glutamyl transferase (GGT) because it is involved in glutathione metabolism. It is mostly produced by the liver, but also in the kidney. GGT is known to regulate apoptotic balance and promotes cancer progression and invasion. Hofbauer et al (18) performed a study on 921 patients diagnosed with RCC, in which the serum levels of GGT were monitored prior to surgical treatment. The authors of this study concluded that pre-operative serum value of GGT is a new and independent prognostic factor. Its value can be used for individual treatment plans, but also for patient counseling and surveillance (18).

The manifestation of a tumor cell requires a mandatory alteration in the natural apoptotic mechanism, and an imbalance between anti-apoptotic and pro-apoptotic proteins leads to tumor proliferation. One of the most important molecules involved in cell apoptosis is the tumor necrosis factor-related apoptosis inducing ligand, or TRAIL, because it is mainly observed in tumor cells rather than normal cells. One of the explanations for this occurrence can be the different expression levels of agonistic receptors in tumor cells and the antagonistic receptors in normal cells. There are two death receptors, TRAIL R1 and TRAIL R2, which are agonist receptors for TRAIL and that are known to have the ability 
to transmit a pro-apoptotic cascade signal which eventually leads to cell death (19). By contrast, another two receptors, termed TRAIL R3 and TRAIL R4, which bind to TRAIL do not induce apoptosis, being antagonist receptors, as they do not have an intercellular death domain. Previous findings have shown that TRAIL which is expressed in immune cells induces apoptosis in RCC cells. Macher-Goeppinger et al conducted a study on 838 patients with RCC in which the expression of TRAIL and TRAIL receptors and their link with this disease was evaluated. Those authors concluded that high TRAIL-R2, high TRAIL, and low TRAIL-R4, are associated with low disease-specific survival in RCC, thus the analysis of these molecules can offer valuable prognostic information regarding the adjuvant therapies that can be used on these patients (20). Toiyama et al performed a study where he compared the TRAIL serum level of 83 patients with RCC, prior to treatment, with the TRAIL serum level of 52 healthy individuals. The TRAIL level was significantly lower in the serum of the RCC patients vs. the healthy group. After surgical treatment the TRAIL level increased, and there was a significantly higher specific survival rate of patients with high serum levels of TRAIL compared with those who had a low level of TRAIL in their serum. Thus, the serum level of TRAIL can be an important biomarker for patients with RCC (21).

Tumor formations are comprised of component fibroblasts and macrophages that are responsible for releasing growth factors and cytokines that allow tumor growth to progress; therefore, tumor mass growth is firmly related to its microenvironment. Hypoxia-inducible transcriptional factors such as HIF- $1 \alpha$ and HIF-1 $\beta$ are activated by hypoxia, and their action is to activate pro-angiogenetic factors such as VEGF (22). The Von Hipple-Lindau (VHL) protein is involved in the stabilization of these HIF factors, and their mutation facilitates the manifestation of RCC. This protein is altered in almost $85 \%$ of all RCC cases. Another hypoxia-induced factor termed prolyl hydroxylase 3 or PHD3 is known to be overexpressed in renal tumors, and it is strictly related to VHL. High levels of these factors in the serum of patients with RCC, compared with healthy individuals, and a decrease in levels following surgical treatment have been reported. Kim et al performed a study on 56 patients diagnosed with RCC that underwent radical or nephron-sparing nephrectomy and 56 healthy control individuals. A blood sample was taken prior to the surgery, as well as 1 and 3 months after the surgery. The serum level of PHD3 was measured with ELISA and compared between the two groups. The serum level was higher in RCC patients than in the control. Over $75 \%$ of patients with RCC had T1a disease, and $78 \%$ had clear cell RCC (ccRCC), but there was no significant difference with the higher stage tumor and different histological type. After surgery, the serum level of PHD3 was decreased by $89 \%$ after 1 month and $84 \%$ after 3 months, meaning that PHD3 is a new and potentially important biomarker for RCC (23).

Carbonic anhydrases, enzymes promoted by hypoxia, are responsible for the acidification of gastric and renal tissues and their alteration has been identified in many tumors. Carbonic anhydrase IX (CA IX) is a new and important marker of tumor hypoxia with considerable potential for becoming a valuable diagnostic biomarker for RCC. This enzyme protein is involved in the conversion of carbon dioxide to bicarbonate and in the ion transport and $\mathrm{pH}$ control (24). Findings have shown that the higher the expression of this protein in lung, breast, and head cancer, the more aggressive the tumor is; but concerning RCC, this protein appears to be an early event and is linked with a better prognosis. Using RT-PCR and ELISA, Takacova et al (25) examined CA IX expression in 74 patients with RCC. The tests came back positive for the expression of CA IX in 24 cases. Most of the positive samples were from patients with clear cell RCC (18 cases); 4 cases from papillary renal cell carcinoma (PRCC) and 2 cases with chromophobe renal cell carcinoma (CHRCC). There were also 4 cases with negative CA IX but with benign tumors. It was also observed that the patients with a high tumor stage (T3) had a lower positivity of CA IX, $17 \%$ compared with the patients with T1 disease who were positive in $50 \%$ of the cases (25).

Other important enzymes that are involved in tumor cell metabolism and proliferation include pyruvate kinase type M2 (TuM2 PK) and thymidine kinase 1 (TK1). The enzymes were studied by Nisman et al (26) in 116 patients with RCC and compared with 20 healthy patients. Those authors followed up the link between the circulating levels of the enzymes preoperatively and disease recurrence. Nisman et al (26) observed that there is a high association between the levels of the two enzymes with tumor stage. The TuM2 PK marker was found to be associated with tumor grade and extensive tumor necrosis (over 50\%). In addition, the recurrence rate of patients with a high level of TuM2 PK and TK1 was higher compared to those patients who had low levels of the two enzymes. Thus, the two biomarkers can be used in patients with RCC prior to nephrectomy to predict recurrence and to redirect them to adjuvant treatment (26).

de Martino et al (27) performed a study on another enzyme involved in RCC relating to the significance of circulating $20 \mathrm{~S}$ proteasome levels. The serum level of this enzyme was obtained by a sandwich enzyme-linked immunosorbent assay. That study included 113 patients with RCC and 15 healthy controls. High levels of $20 \mathrm{~S}$ proteasome were observed in patients with RCC associated with high Fuhrman grade, metastasis, tumor necrosis and $\mathrm{T}$ stage. In addition, tumor size was correlated with high levels of this enzyme. There was no association with sex, age or $\mathrm{N}$ stage. The risk of death from renal tumor was found to be increased by $21 \%$ with each $1 \mathrm{mg}$ increase in the $20 \mathrm{~S}$ proteasome level (27).

Over the years, scientists have researched aspects of RNA and DNA involved with the manifestation of tumor cells. In recent years, the role of circular RNA (circRNA) in oncology has increased in popularity, mainly because many studies have reported that multiple circRNAs are involved in cancer cell functions and can also serve as a prognostic factor in patients with cancer (28). A new circular RNA called circ-ABCB10, was shown to have an important impact on patients with RCC. Huang et al performed a study in which the effect of circ-ABCB10 was evaluated on the proliferation and apoptosis of ccRCC, and its prognostic value. Their study focused on measuring the circ-ABCB12 expression in five ccRCC cell lines and in normal kidney using quantitative polymerase chain reaction in 120 patients with ccRCC. The apoptotic markers were measured using western blot analysis. Authors of that study found that circ-ABCB10 expression was elevated in all the patients with RCC, compared with the heathy patients. The 
cell line A498, was rich with circ-ABCB10 in the ccRCC cell lines but was poor with circ-ABCB10 in normal kidney cell lines In addition, A498 cell apoptosis was highly represented with circABCB10 compared with the healthy kidney cell lines. Authors of that study concluded that circ-ABCB10 high expression was linked with the advanced pathological grade and tumor metastasis stage. It was also found to be an independent predictive factor for a worse survival in RCC patients regarding overall survival (28).

MicroRNAs. MicroRNAs (miRNAs/miRs) are well known to play important roles in cancer progression, carcinogenesis and invasion. Heinemann et al performed a study designed to identify microRNAs as potential biomarkers for RCC. The study included 18 patients with ccRCC and 8 patients with benign renal tumors. Authors of that study determined the serum small RNA expression profile of patients using small RNA sequencing. This study successfully identified high values of serum miR-122-5p and miR-206 in the patients with RCC, compared with healthy individuals. It was also observed that the value of these two microRNAs were at similar levels in both RCC and benign renal tumor patients. High values of miR-122-5p and miR-206 were directly associated with metastatic RCC and pT stage, and also with a shorter period of progression-free and overall survival in these patients (29).

\section{Urine RCC biomarkers}

In addition to the RCC biomarkers that exist in the blood of patients, there are some markers that exist in the urine thereof. One of these markers is nuclear matrix protein 22 (NMP22), which is part of the internal framework of the nucleus. It is involved in DNA replication and its characteristics render it a good biomarker of tumor cells. NMP22 was identified to be strictly linked to transitional cell carcinoma (30). In addition, results of this study showed increased levels of NMP22 in patients with RCC; therefore, NMP22 could be used as an RCC screening marker.

NGAL or neutrophil gelatinase-associated lipocalin was demonstrated to be a sensitive biomarker for tubular injury, but can also be expressed by several histological types of renal tumors. In this case the presence of NGAL can be found in patients with renal cancer and indicates a highly elevated histological grade of ccRCC and papillary RCC. Its expression in urine is most likely linked with the tubular injury produced by the tumor. In a previous study, Di Carlo demonstrated a high level of NGAL in patients with RCC, compared with healthy, normal individuals (31).

Other proteins present in the urine of patients that have undergone surgical excision of an RCC tumor, aquaporin-1 (AQP1) and perilipin 2 (PER2), respectively, can be used as a screening method and surveillance, mainly because they are not significantly influenced by noncancerous kidney diseases, including infections, or glomerulonephritis of other nephropathies (32). Morrissey et al performed a study on AQP1 and adipophilin (ADFP) proteins that can be found in the urine of patients. The aim of the study was to determine the effectiveness of these proteins regarding RCC and other non-oncological kidney injuries. For this, the study included 36 patients with clear cell or papillary kidney cancer,
43 controls, 44 patients with documented urinary tract infection, 24 patients diagnosed with diabetic nephropathy, and 18 patients diagnosed with glomerulonephritis. This study found that the urine concentration of these 2 markers were 23-fold higher regarding AQP1, respectively, 4-fold higher regarding ADFP, compared to healthy persons. Additionally, there was a direct link between the tumor size and value of these markers. Regarding the correlation between AQP1 and ADFP and other common kidney disease, the results showed that their value was not as increased as in the cases of the patients with kidney cancer. In conclusion, that study related that the urine concentration of AQP1 and ADFP is highly correlated with RCC, and common kidney disease has little impact on the value of these markers, as found in the case of malignant tumors (32).

\section{Other useful biomarkers for RCC patients}

A new transmembrane protein believed to be responsible for triggering the extrinsic coagulation pathway known as tissue factor (TF) promises to be another important serum biomarker regarding patients with RCC. It is known that a high level of $\mathrm{TF}$ is linked to negative outcomes of varied tumors of the lung, breast, prostate, and even the nervous system (33). TF is also identified in immunohistochemical tests in Wilms' tumor and in clear cell carcinoma (33). However, Silva et al (34) researched whether the serum TF level can be used as a biomarker for RCC. The study included 30 patients with ccRCC and 16 controls with other pathologies, treated surgically. Prior to surgery, the value of TF was verified from the blood of the patients by ELISA test, using the Human Coagulation FactorIII/Tissue Factor Immunoassay-Quantikine ${ }^{\circledR}$ ELISA kit. In addition, authors of that study observed that the mean value of TF in the serum of the patients with RCC was $66.8 \mathrm{pg} / \mathrm{dl}$, whereas the mean value of TF in the blood of the control group was only $28.4 \mathrm{pg} / \mathrm{dl}$. After surgery, the authors observed a decrease of TF in the serum of the patients, with a mean value of $41.6 \mathrm{pg} / \mathrm{dl}$. This second blood test was performed at least four weeks after surgery. The purpose of this study was also to determine which feature is involved in increasing the serum level of TF (tumor size, TNM classification and Fuhrman grade, or the presence of necrosis). The only variable that had a positive association was tumor size. Thus, those authors concluded that with each cm increase in tumor size, the value of TF increased approximately $4.7 \mathrm{pg} / \mathrm{dl}$. Thus, the value of TF in the serum of patients with RCC can be used as a reliable biomarker, as it is correlated with tumor size (34).

One of the main roles of the kidney is to purify the blood from nitrogenous substances and to regulate the levels of amino acids from plasma. A normal renal tube is able to reabsorb the amino acids, and because RCC derives from renal tube cells, there is a possibility that these tumor types determine an alteration of the tube ability to reabsorb amino acids; therefore, they may be present in the serum of patients. Additionally, growth of a renal tumor requires a high concentration of amino acids such as methionine, glutamine, and asparagine (35). Extensive research has been conducted to determine whether serum amino acid levels are a possible biomarker for RCC. One such study was conducted by Mustafa et al on 189 patients diagnosed with renal carcinoma, and 104 controls. The aim of 
this study was to examine the concentrations of 26 different amino acids in patient serum, before surgical treatment. From all 26 amino acids studied, only 15 amino acids showed relevance between the control and patients with RCC. From the 15 amino acids, 13 were decreased in patients with RCC compared to the control, and 2 amino acids, arginine and cysteine, were increased. A substantial difference between the control and the RCC patients from the lower-level amino acids was observed for histidine and omithine. As many amino acids were altered, the focus of the study was on the strongest correlation with each of the groups. The strongest correlation was between valine, leucine and isoleucine. Based on the tumor grade and type, the study concluded that there was no significant difference in the concentration of amino acids in the serum of patients regarding clear cell, and papillary type of tumor. Additionally, early stage tumors (stage 1 and 2) had a slightly lower model score than large tumors, but in both cases compared with the control, the levels were elevated. Overall, it was concluded that there is an alteration regarding 15 types of amino acids for both early and late stage of patients with RCC. Moreover, the level of amino acids in the serum of patients has a predictive value in overall survival, and also in recurrence, and therefore may be used as a detection and prognostic factor for RCC (36).

Other biomarkers such as hepatic hormone hepcidin and growth/differentiation factor 15 (GDF15), usually used in other cancer types including breast, colon and lung cancer, were researched to be used as biomarkers for RCC. The hepatic hormone hepcidin is the main regulator of iron homeostasis. A high value of hepcidin in the serum is usually associated with metastatic disease and poor outcome for prostate and breast cancer, as well as for renal cell carcinoma. GDF15 plays an important role regarding tissue regeneration and is increased in various types of cancer (37). Traeger et al aimed to find a link between hepcidin and GDF15 and RCC and urothelial carcinoma of the upper urinary tract. In total, 94 patients with RCC and 21 controls were involved in this study. The serum level of GDF15 and hepcidin were measured for each patient. From the 94 patients, 44 patients presented metastatic disease; therefore, all 94 patients were divided in 2 groups: Metastatic and non-metastatic disease. The serum level of hepcidin and GDF15 was high in the two groups. The non-metastatic group had a lower level of GDF15 compared with the metastatic group. GDF15 was also increased in patients who presented relapse, compared with the control group. Patients with RCC who presented relapse presented a high level of hepcidin compared with both the control and no relapse group. The study also demonstrated that the overall survival of patients with RCC was decreased when the hepcidin level was higher than $12.7 \mathrm{ng} / \mathrm{dl}$, and/or the GDF15 level was higher than 1,200 pg/dl. Thus, a high value of both biomarkers was found to be associated with metastatic RCC as well as a high incidence of relapse (37).

\section{Discussion}

The management of patients diagnosed with renal tumors remains a problem regarding the means of early diagnosis, despite available studies on suitable biomarkers that can be used as a screening method. There is a need of alternative therapy besides classic surgery. Furthermore, the earlier the disease is detected the lower the morbidity.

One of the most studied surface antigens is represented by CA IX, and recent studies have demonstrated that the blood level of CA IX can be used for diagnosis of RCC, because it is an important marker of tumor hypoxia. The level of CA IX in patient serum can be used for optimal management of patients with RCC, in particular for monitoring the progression and even the recurrence of the disease. In order to be fully used as a reliable RCC biomarker, additional studies should be performed on many patients along with relevant follow-up.

The accumulation of HSP27 is promoted by shear stress, in order to regulate cytoskeleton dynamics. It also promotes depolymerization into small molecules (15). All of this transformation suggests that this protein is crucial in tumor cell migration; therefore it can be used as a biomarker for renal tumors.

Recent studies have demonstrated that preoperative $20 \mathrm{~S}$ proteasome plays an important role due to its increased levels in patients with RCC. This important biomarker was first mentioned by Lavabre-Bertrand et al in the serum of patients suffering from various types of cancer (38-40). de Martino et al (27) studied this biomarker in patients with ccRCC, with good results regarding the possibility of being used as a predictive, diagnostic and prognostic marker.

With the development of new technologies, the understanding of RCC biology has increasingly improved. This development has led to new systemic therapies that have improved patient quality of life. All research demonstrated that the manifestation of RCC is partly driven by the HIF factor protein, VHL gene and the ubiquitin pathway. These are also responsible for the progression of ccRCC.

\section{Conclusions}

In conclusion, there are several biomarkers present in the blood and urine of patients with RCC that may be used for screening, detection and prognosis of RCC. HSP27, amyloid A, GGT, TRAIL, VHL protein, PHD3, CA IX, TuM2 PK, TK1, 20S proteasome, circ-ABCB10, miR-206, miR-122-5p, NGAL, AQP1 and PER2, TF, hepcidin and GDF15, are all important biomarkers whose levels are high in RCC and may play an important role in the future for early diagnosis, prognosis and overall survival for these patients $(41,42)$. Nevertheless, more studies should be performed on these molecules to confirm their benefit.

\section{Acknowledgements}

Not applicable.

\section{Funding}

No funding was received.

\section{Availability of data and materials}

All information provided in this review is documented by relevant references. 


\section{Authors' contributions}

OB, RA had the initial idea for the project, developed the project, wrote the manuscript and supervised. DaM, DS, $\mathrm{IB}, \mathrm{CoS}$ and NB developed the project, collected the data and references and were major contributors in writing the manuscript. LI, AC, DrM, CaS and CD collected the data and wrote the manuscript. NB and CD provided consultation to the manuscript and supervised the project. All authors read and approved the final manuscript.

\section{Ethics approval and consent to participate}

Not applicable.

\section{Patient consent for publication}

Not applicable.

\section{Competing interests}

The authors declare that they have no competing interests.

\section{References}

1. Lipworth L, Tarone RE and McLaughlin JK: The epidemiology of renal cell carcinoma. J Urol 176: 2353-2358, 2006.

2. Eichelberg C, Junker K, Ljungberg B and Moch H: Diagnostic and prognostic molecular markers for renal cell carcinoma: A critical appraisal of the current state of research and clinical applicability. Eur Urol 55: 851-863, 2009.

3. Ljungberg B, Bensalah K, Canfield S, Dabestani S, Hofmann F, Hora M, Kuczyk MA, Lam T, Marconi L, Merseburger AS, et al: EAU guidelines on renal cell carcinoma: 2014 update. Eur Urol 67: 913-924, 2015.

4. Landolt L, Eikrem O, Strauss P, Scherer A, Lovett DH, Beisland C, Finne K, Osman T, Ibrahim MM, Gausdal G, et al: Clear cell renal cell carcinoma is linked to epithelial-to-mesenchymal transition and to fibrosis. Physiol Rep 5: e13305, 2017.

5. Grivennikov SI, Greten FR and Karin M: Immunity, inflammation, and cancer. Cell 140: 883-899, 2010.

6. Vesely MD, Kershaw MH, Schreiber RD and Smyth MJ: Natural innate and adaptive immunity to cancer. Annu Rev Immunol 29: 235-271, 2011

7. Linehan WM, Vasselli J, Srinivasan R, Walther MM, Merino M, Choyke P, Vocke C, Schmidt L, Isaacs JS, Glenn G, et al: Genetic basis of cancer of the kidney: Disease-specific approaches to therapy. Clin Cancer Res 10 6282S-6289S, 2004.

8. Rajandram R, Yap NY, Pailoor J, Razack AH, Ng KL, Ong TA, Morais C and Gobe GC: Tumour necrosis factor receptor-associated factor-1 (TRAF-1) expression is increased in renal cell carcinoma patient serum but decreased in cancer tissue compared with normal: Potential biomarker significance. Pathology 46: 518-522, 2014

9. Gougos A and Letarte M: Primary structure of endoglin, an RGD-containing glycoprotein of human endothelial cells. J Biol Chem 265: 8361-8364, 1990.

10. Bussolati B, Bruno S, Grange C, Ferrando U and Camussi G: Identification of a tumor-initiating stem cell population in human renal carcinomas. FASEB J 22: 3696-3705, 2008.

11. Saroufim A, Messai Y, Hasmim M, Rioux N, Iacovelli R, Verhoest G, Bensalah K, Patard JJ, Albiges L, Azzarone B, et al: Tumoral CD105 is a novel independent prognostic marker for prognosis in clear-cell renal cell carcinoma. Br J Cancer 110: 1778-1784, 2014.

12. Kampinga HH, Hageman J, Vos MJ, Kubota H, Tanguay RM, Bruford EA, Cheetham ME, Chen B and Hightower LE: Guidelines for the nomenclature of the human heat shock proteins. Cell Stress Chaperones 14: 105-111, 2009.
13. Lianos GD, Alexiou GA, Mangano A, Mangano A, Rausei S, Boni L, Dionigi G and Roukos DH: The role of heat shock proteins in cancer. Cancer Lett 360: 114-118, 2015.

14. Zhang B, Xie F, Aziz AUR, Shao S, Li W, Deng S, Liao X and Liu B: Heat shock protein 27 phosphorylation regulates tumor cell migration under shear stress. Biomolecules 9: 50, 2019.

15. White NM, Masui O, Desouza LV, Krakovska O, Metias S, Romaschin AD, Honey RJ, Stewart R, Pace K, Lee J, et al: Quantitative proteomic analysis reveals potential diagnostic markers and pathways involved in pathogenesis of renal cell carcinoma. Oncotarget 5: 506-518, 2014.

16. de Vivar Chevez AR, Finke J and Bukowski R: The role of inflammation in kidney cancer. Adv Exp Med Biol 816: 197-234, 2014.

17. Fischer K, Theil G, Hoda R and Fornara P: Serum amyloid A: A biomarker for renal cancer. Anticancer Res 32: 1801-1804, 2012.

18. Hofbauer SL, Stangl KI, de Martino M, Lucca I, Haitel A, Shariat SF and Klatte T: Pretherapeutic gamma-glutamyltransferase is an independent prognostic factor for patients with renal cell carcinoma. Br J Cancer 111: 1526-1531, 2014.

19. Pan G, O'Rourke K, Chinnaiyan AM, Gentz R, Ebner R, Ni J and Dixit VM: The receptor for the cytotoxic ligand TRAIL. Science 276: 111-113, 1997.

20. Macher-Goeppinger S, Aulmann S, Tagscherer KE, Wagener N, Haferkamp A, Penzel R, Brauckhoff A, Hohenfellner M, Sykora J, Walczak H, et al: Prognostic value of tumor necrosis factor-related apoptosis-inducing ligand (TRAIL) and TRAIL receptors in renal cell cancer. Clin Cancer Res 15: 650-659, 2009.

21. Toiyama D, Takaha N, Shinnoh M, Ueda T, Kimura Y, Nakamura T, Hongo F, Mikami K, Kamoi K, Kawauchi A and Miki T: Significance of serum tumor necrosis factor-related apoptosis-inducing ligand as a prognostic biomarker for renal cell carcinoma. Mol Clin Oncol 1: 69-74, 2013.

22. Frew IJ and Moch H: A clearer view of the molecular complexity of clear cell renal cell carcinoma. Annu Rev Pathol 10: 263-289, 2015.

23. Kim KH, Lee HH, Yoon YE, Na JC, Kim KS and Han WK: Prolyl hydroxylase-3 is a novel renal cell carcinoma biomarker. Investig Clin Urol 60: 425-431, 2019.

24. Svastova E, Hulikova A, Rafajova M, Zat'ovicova M, Gibadulinova A, Casini A, Cecchi A, Scozzafava A, Supuran CT, Pastorek J and Pastoreková S: Hypoxia activates the capacity of tumor-associated carbonic anhydrase IX to acidify extracellular pH. FEBS Lett 577: 439-445, 2004.

25. Takacova M, Bartosova M, Skvarkova L, Zatovicova M, Vidlickova I, Csaderova L, Barathova M, Breza J Jr, Bujdak P, Pastorek J, et al: Carbonic anhydrase IX is a clinically significant tissue and serum biomarker associated with renal cell carcinoma. Oncol Lett 5: 191-197, 2013.

26. Nisman B, Yutkin V, Nechushtan H, Gofrit ON, Peretz T, Gronowitz S and Pode D: Circulating tumor M2 pyruvate kinase and thymidine kinase 1 are potential predictors for disease recurrence in renal cell carcinoma after nephrectomy. Urology 76: 513.e1-6, 2010.

27. de Martino M, Hoetzenecker K, Ankersmit HJ, Roth GA, Haitel A, Waldert M and Klatte T: Serum 20S proteasome is elevated in patients with renal cell carcinoma and associated with poor prognosis. Br J Cancer 106: 904-908, 2012.

28. Huang Y,Zhang Y, Jia L, Liu C and Xu F: Circular RNA ABCB10 promotes tumor progression and correlates with pejorative prognosis in clear cell renal cell carcinoma. Int J Biol Markers 34: 176-183, 2019.

29. Heinemann FG, Tolkach Y, Deng M, Schmidt D, Perner S, Kristiansen G, Muller SC and Ellinger J: Serum miR-122-5p and miR-206 expression: Non-invasive prognostic biomarkers for renal cell carcinoma. Clin Epigenetics 10: 11, 2018.

30. Di Carlo A, Terracciano D, Mariano A, Oliva A, D'Armiento M and Macchia V: Role of cytokeratins, nuclear matrix proteins, Lewis antigen and epidermal growth factor receptor in human bladder tumors. Int J Oncol 23: 757-762, 2003.

31. Di Carlo A: Evaluation of neutrophil gelatinase-associated lipocalin (NGAL), matrix metalloproteinase-9 (MMP-9) and their complex MMP-9/NGAL in sera and urine of patients with kidney tumors. Oncol Lett 5: 1677-1681, 2013.

32. Morrissey JJ and Kharasch ED: The specificity of urinary aquaporin 1 and perilipin 2 to screen for renal cell carcinoma. J Urol 189: 1913-1920, 2013.

33. Kasthuri RS, Taubman MB and Mackman N: Role of tissue factor in cancer. J Clin Oncol 27: 4834-4838, 2009.

34. Silva DD, Noronha JAP, Pinheiro da Costa BE, Zandona PCE and Carvalhal GF: Serum tissue factor as a biomarker for renal clear cell carcinoma. Int Braz J Urol 44: 38-44, 2018. 
35. Stern PH, Wallace CD and Hoffman RM: Altered methionine metabolism occurs in all members of a set of diverse human tumor cell lines. J Cell Physiol 119: 29-34, 1984.

36. Mustafa A, Gupta S, Hudes GR, Egleston BL, Uzzo RG and Kruger WD: Serum amino acid levels as a biomarker for renal cell carcinoma. J Urol 186: 1206-1212, 2011.

37. Traeger L, Ellermann I, Wiethoff H, Ihbe J, Gallitz I, Eveslage M, Moritz R, Herrmann E, Schrader AJ and Steinbicker AU: Serum Hepcidin and GDF-15 levels as prognostic markers in urothelial carcinoma of the upper urinary tract and renal cell carcinoma. BMC Cancer 19: 74, 2019.

38. Lavabre-Bertrand T, Henry L, Carillo S, Guiraud I, Ouali A, Dutaud D, Aubry L, Rossi JF and Bureau JP: Plasma proteasome level is a potential marker in patients with solid tumors and hemopoietic malignancies. Cancer 92: 2493-2500, 2001.
39. Bratu OG, Cherciu AI, Bumbu A, Lupu S, Marcu DR, Ionita-Radu F, Manea M, Furau C, Diaconu CC and Mischianu DLD: Retroperitoneal tumours-treatment and prognosis of tumour recurrence. Rev Chim 70: 190-194, 2019.

40. Marcu DR, Ionita-Radu F, Iorga LD, Manea M, Socea B, Scarneciu I, Isvoranu G, Costache R, Diaconu CC and Bratu OG: Vascular involvement in primary retroperitoneal tumors. Rev Chim (Bucharest) 70: 445-448, 2019.

41. Boda D: Cellomics as integrative omics for cancer. Curr Proteomics 10: 237-245, 2013.

42. Neagu M, Constantin C, Tanase C and Boda D: Patented biomarker panels in early detection of cancer. Recent Patents Biomarkers 1: 10-24, 2011. 